\title{
A Semi-Group Theoretical Analysis of a Finite Element Method for a Linearized Viscous Shallow-Water System
}

\author{
By
}

\author{
Teruo UshiJima*
}

\begin{abstract}
A finite element step-by-step time integration scheme of a model linear viscous shallowwater system is analyzed by the approximation theory for semi-groups of linear operators. Strong convergence and $O(h+\tau)$ error estimate in $L^{2}$-sense are established.
\end{abstract}

\section{Introduction}

The aim of this paper is to present a semi-group theoretical proof of convergence of finite element approximate solutions of a model linear system arising in the study of viscous shallow-water equations.

It is widely recognized that Trotter-Kato approximation therorem for continuous semi-groups ([16], [8], [9]) and its variants are powerful tools to give mathematical analysis of numerical method applied to evolution problems if the finite element approximation is employed as the approximation method with respect to space variables. In author's previous works [17], [18], and [19], this semi-group theoretical method was developed and applied to the 2 nd order linear hyperbolic equation with time independent coefficients. For parabolic equations, Fujita-Mizutani [3] presented excellent error estimates using the Dunford integral technique. There are several applications and extensions of this general framework. Here we list some examples of such works done in Japan. Fujita [2] and Suzuki [14] treated the case of linear parabolic equations with time dependent coefficients, and Okamoto [12] treated Stokes equation. Kikuchi-Ushijima [10] discussed artificial viscosity technique applied to the 1 st

Received January 5, 1983.

* Department of Information Mathematics, The University of Electro-Communications, 1-5-1, Chofugaoka, Chofu-shi, Tokyo 182, Japan. 
order linear hyperbolic equation. As for viscous shallow-water equation, which is a typical example of incomplete parabolic system, a linear model is analyzed in Kanayama-Ushijima [5], [6].

This work is closely related to the previous works [5], [6] and [7], in which our present model system is derived. In [6], we discussed about a slightly artificial time discretization method. In the present work, we investigate a time discretization method which is very similar to the practical computational method used in Kanayama-Ohtsuka [4].

In Section 1 of this paper, the approximation theory for semi-group of linear operators is summarized with emphasis on the application to finite element method. In Section 2, our continuous model system is described, and solved with the aid of the theory of contraction semi-group of linear operators acting on a Hilbert space. In Section 3, a finite element scheme is presented and main results are stated in Theorems 3.1 and 3.2. Section 4 is devoted to prove these theorems. In Section 5, an $O(h+\tau)$ error estimate in $L^{2}$-norm is obtained under the assumption of the existence of sufficiently smooth classical solution of the original model equations.

Now we explain our notations and terminologies. The totality of continuous linear operators from a Banach space $X$ into a Banach space $Y$ is denoted by $L(X, Y)$, and $L(X, X)$ by $L(X)$, the domain, the range, the null space, and the resolvent set of a linear operator $A$ by $D(A), R(A), N(A)$, and $\rho(A)$, respectively. Norms of various Banach spaces are frequently denoted by the same symbol \| $\|$, and sometimes by, for example, \|\|$_{X}$ when we need specifications. We simply call a semi-group of class $C_{0}$ (see [20], [9], [11]) a continuous semi-group in this paper.

The author would like to express his sincere thanks to Mr. H. Kanayama of FUJIFACOM Corporation, who gave the author the concrete knowledge of the viscous shallow-water equations, through a series of joint researches, and to Professor H. Fujita of the University of Tokyo, who gave the author the constant encouragement with valuable discussions and suggestions during the whole period of preparation of this work. The author is highly grateful to the referee of this paper for his constructive comment. 


\section{§1. Some Results from the Approximation Theory for Semi-Groups of Linear Operators}

Let $X$ be a real Banach space, and let $\left\{X_{h}: 0<h \leq \bar{h}\right\}$ be a family of real Banach spaces. We say that $X_{h}$ converges to $X$ as $h$ tends to 0 in the sense of Kato (in abbreviation, we write $X_{h} \stackrel{K}{\longrightarrow} X$ ) if for any $h$ there is a linear operator $P_{h} \in L\left(X, X_{h}\right)$ (called an approximating operator) satisfying the following three conditions:

(K.1) $\lim _{h \rightarrow 0}\left\|P_{h} x\right\|=\|x\| \quad$ for any $\quad x \in X$.

(K.2) $\left\{\begin{array}{l}\text { Each } x_{h} \in X_{h} \text { can be expressed as } x_{h}=P_{h} x^{(h)} \text { with } x^{(h)} \in X, \text { and there } \\ \text { is a constant } N \text { independent of } h \text { such that }\left\|x^{(h)}\right\| \leq N\left\|x_{h}\right\| .\end{array}\right.$

(K.3) There is a constant $N^{\prime}$ independent of $h$ such that $\left\|P_{h}\right\| \leq N^{\prime}$.

Now we fix a family $\left\{X_{h}\right\}$ and a space $X$ such that $X_{h} \stackrel{K}{\longrightarrow} X$. Then a sequence $\left\{x_{h}: x_{h} \in X_{h}\right\}$ is said to $K$-converge to $x \in X$ if $\lim _{h \rightarrow 0}\left\|x_{h}-P_{h} x\right\|=0$. And a sequence $\left\{A_{h}: A_{h} \in L\left(X_{h}\right)\right\}$ is said to $K$-converge to $A \in L(X)$ (in abbreviation, we write $A_{h} \stackrel{K}{\longrightarrow} A$ ) if $A_{h} P_{h} x K$-converges to $A x$ for any $x \in X$, or equivalently

$$
\lim _{h \rightarrow 0}\left\|A_{h} P_{h} x-P_{h} A x\right\|=0 \quad \text { for any } \quad x \in X .
$$

Let $\Lambda$ be an index set. Sequences $\left\{A_{\lambda h} \in L\left(X_{h}\right)\right\}_{\lambda \in A}$ are said to $K$-converge to $A_{\lambda} \in L(X)$ uniformly in $\lambda \in \Lambda$ if for any $x \in X$

$$
\lim _{h \rightarrow 0}\left\|A_{\lambda h} P_{h} x-P_{h} A_{\lambda} x\right\|=0
$$

holds uniformly in $\lambda \in \Lambda$.

Let $A_{h}$, and $A$ be the generator of a continuous semi-group $T_{h}(t) \in L\left(X_{h}\right)$, and $T(t) \in L(X)$, respectively. Consider the following three conditions.

(A) $\left\{\begin{array}{l}\text { (Consistency). There is a real number } \lambda \text { contained in } \rho\left(A_{h}\right)(0<h \leq \bar{h}), \\ \text { and in } \rho(A) \text { such that }\left(\lambda-A_{h}\right)^{-1} \stackrel{K}{\longrightarrow}(\lambda-A)^{-1} .\end{array}\right.$
(B) $\left\{\begin{array}{l}\text { (Boundedness). There are positive numbers } T \text { and } M \text { such that }\left\|T_{h}(t)\right\| \\ \leq M \text { for any } t \in[0, T] \text { and } h \in(0, \bar{h}] .\end{array}\right.$
(C) $\left\{\begin{array}{l}\text { (Convergence). For any finite } T, T_{h}(t) \stackrel{K}{\longrightarrow} T(t) \text { holds uniformly in } \\ t \in[0, T] .\end{array}\right.$

Then we have the following fundamental theorem. 
Theorem $1.1(\mathrm{~A}-\mathrm{B}-\mathrm{C}$ Theorem). The conditions (A) and (B) hold if and only if $(\mathrm{C})$ does.

The proof of this theorem can be obtained by a suitable interpretation of the well-known approximation theory of semi-groups of linear operators due to Trotter and Kato (See [16], [8] and [9]). In the author's previous work [17], $\mathrm{A}-\mathrm{B}-\mathrm{C}$ theorem is proved in the setting of the notion of $K$-convergence with respect to a countable suffix " $n$ ", which corresponds to the continuous suffix $h$ in the present setting. As noted in [17], if $h$ is a countable suffix tending to zero, (K.3) follows from (K.1) and (K.2). In the present formulation, however, we prefer the continuous suffix $h$ which well suits the custom of finite element analysis. Hence we require the condition (K.3).

Let $\tau>0$. An $L(X)$-valued function $T(t)$ defined on $[0, \infty)$ is said to be a discrete semi-group with time unit $\tau$ if $T(t)$ can be expressed as $T(t)=T(\tau)^{[t / \tau]}$ for any $t \geq 0$ with an operator $T(\tau) \in L(X)$, where $[\alpha]$ denotes the greatest integer being less than or equal to the real number $\alpha$. The generator $A$ of such a semigroup is defined by $A=\frac{T(\tau)-1}{\tau} \in L(X)$.

Now we can consider the approximation for the continuous semi-group $T(t) \in L(X)$ with the generator $A$ by the discrete semi-group $T_{h}(t) \in L\left(X_{h}\right)$ with the generator $A_{h} \in L\left(X_{h}\right)$. Let $\tau_{h}$ be the time unit of $T_{h}(t)$. Under the assumption

$$
\lim _{h \rightarrow 0} \tau_{h}=0,
$$

let us consider the conditions (A), (B) and (C) given as above for the present $A_{h}, A, T_{h}(t)$ and $T(t)$. Then Theorem 1.1 remains valid. Namely we have

Theorem 1.2 (Discrete version of A-B-C theorem). Under the assumption (T), the conditions (A) and (B) hold if and only if (C) does.

The proof of this Theorem can be found in [17] at least in the case of countable suffix " $n$ " instead of continuous suffix $h$.

Now we add a remark on the relation between discrete version of $A-B-C$ theorem and Equivalence theorem due to Lax (cf. Richtmeyer-Morton [13]). Consider the following condition:

(D) $\left\{\begin{array}{l}\text { There is a linear subset } D \text { of } D(A) \text {, being dense in } D(A) \text { with respect to } \\ \text { the graph norm: }\|x\|_{D(A)}=\|x\|+\|A x\| \text {, with the property that for any } \\ x \in D, A_{h} P_{h} x \quad K \text {-converges to } A x .\end{array}\right.$ 
From the conditions (B) and (T), we can conclude that there are constants $M^{\prime}$ and $\omega$ independent of $h$ such that

$$
\left\|e^{t A h}\right\| \leq M^{\prime} e^{t \omega} \quad \text { for any } t \geq 0 .
$$

This estimate (1.1) implies that

$$
\left\|\left(\lambda-A_{h}\right)^{-1}\right\| \leq M^{\prime}(\lambda-\omega)^{-1} \text { for } \lambda>\omega .
$$

Using the condition (D) and the estimate (1.2), we can establish the condition (A). Hence we have the following assertion from Theorem 1.2.

Theorem 1.3 (An equivalence theorem). Under the assumptions ( $\mathrm{T})$ and (D), the boundedness condition (B) is equivalent to the convergence condition (C).

\section{§2. A Model Linear System Derived from the Viscous Shallow-Water Theory}

Let $\Omega$ be an open bounded domain in the plane $R^{2}$ with $C^{\infty}$-class boundary $\Gamma$. Assume that we have a two dimensional vector field $b=\left(b_{i}(x)\right)_{i=1,2}$ and a scalar function $H=H(x)$ defined on the closure $\bar{\Omega}$ of $\Omega$ with the following propcrties:

$$
\begin{array}{ll}
b_{i}(x) \quad(i=1,2), & H(x) \in C^{\infty}(\bar{\Omega}) . \\
b(x)=0 \quad \text { for } \quad x \in \Gamma .
\end{array}
$$

(2.3) There are positive constants $\underline{H}$ and $\bar{H}$ such that

$$
\begin{aligned}
& 0<\underline{H} \leq H(x) \leq \bar{H} \quad \text { for } \quad x \in \Omega . \\
& \operatorname{div}(H b)=0 .
\end{aligned}
$$

Here and hereafter we use the notation

$$
\operatorname{div}(v)=\frac{\partial v_{1}}{\partial x_{1}}+\frac{\partial v_{2}}{\partial x_{2}}, \quad \operatorname{grad} \quad q=\left(\frac{\partial q}{\partial x_{i}}\right)_{i=1,2}
$$

for a two dimensional vector field $v=v(x)$, and for a scalar function $q=q(x)$.

A physical explanation of $\Omega, b$ and $H$ are:

$\Omega$ is the horizontal cross section of a lake surrounded by a precipitous cliff, $b$ is a stationary velocity of the water being averaged vertically in a sense, $H$ is a stationary distance between the surface, and the bottom, of the lake.

Wc take the following linear evolution problem as a model system describing the small variation from the stationary state $\{b, H\}$. 
(ஜீ)

$$
\left\{\begin{array}{l}
\frac{\partial u}{\partial t}+(b, \nabla) u=-g \cdot \operatorname{grad} p+\frac{v}{H}(\nabla, H \nabla) u-[f] u-\frac{g_{1}}{H}|b| u, t>0, x \in \Omega, \\
\frac{\partial p}{\partial t}+\operatorname{div}(H u)=0, \quad t>0, x \in \Omega \\
u=0, \quad t>0, x \in \Gamma \\
u=u^{0}(x), \quad t=0, x \in \Omega \\
p=p^{0}(x), \quad t=0, x \in \Omega
\end{array}\right.
$$

Here the 2-vector function $u=\left(u_{i}(t, x)\right)_{i=1,2}$ represents the variation of the velocity from the stationary velocity $b$, and $p=p(t, x)$ represents that of the water surface from the stationary height $H$. The first equation comes from the equation of motion, and the second comes from the conservation law of mass. The real constant $f$, and positive constants, $g, v$ and $g_{1}$ represent the Coriolis constant, the acceleration of gravity, the eddy viscosity coefficient, and $g / C^{2}$ with Chezy's coefficient $C$, respectively. Differential operators $(b, \nabla)$ and $(\nabla, H \nabla)$ are defined by

$$
\begin{aligned}
& (b, \nabla)=\sum_{i=1}^{2} b_{i} \frac{\partial}{\partial x_{i}} \\
& (\nabla, H \nabla)=\sum_{i=1}^{2} \frac{\partial}{\partial x_{i}}\left(H \frac{\partial}{\partial x_{i}}\right)
\end{aligned}
$$

which operate componentwise on 2-vector function $v$. The operator $[f]$ is the matrix multiplication operator defined by the matrix $[f]=\left(\begin{array}{rr}0 & -f \\ f & 0\end{array}\right)$, and the Euclidean length of vector $b$ is denoted by $|b| .\left(|b|=\sqrt{b_{1}^{2}+b_{2}^{2}}\right)$. (As for the derivation of $(\mathscr{E})$ from Reynolds equations, see [5] and [6], where the surface elevation is denoted by $\zeta$ instead of $p$ ).

In order to treat $(\mathscr{E})$ in the frame work of semi-group theory due to HilleYosida, firstly we prepare function spaces. Let $X$ be the totality of 2-vector functions $v=\left(v_{i}\right)_{i=1,2}$ whose components are square integrable over $\Omega$. Define the inner product of $X$ by

$$
(u, v)_{X}=(H u, v)_{\left\{L^{2}(\Omega)\right\}^{2}}=\sum_{i=1}^{2} \int_{\Omega} H u_{i} v_{i} d x .
$$

Then $X$ is a Hilbert space by (2.3). Let $Q$ be $L^{2}(\Omega)$ with the inner product:

$$
(p, q)_{Q}=g(p, q)_{L^{2}(\Omega)}=g \int_{\Omega} p q d x .
$$

Let $X$ be the product Hilbert space $X \times Q$, whose generic element is denoted by $v=\left(\begin{array}{l}v \\ q\end{array}\right), v \in X, q \in Q$. Then the inner product of $X$ is represented by 


$$
(\boldsymbol{u}, \boldsymbol{v})=(u, v)_{X}+(p, q)_{Q} \quad \text { for } \quad \boldsymbol{u}=\left(\begin{array}{l}
u \\
p
\end{array}\right), \boldsymbol{v}=\left(\begin{array}{l}
v \\
q
\end{array}\right) \in \boldsymbol{X} .
$$

Secondly we define bilinear forms, $a(u, v), l(u, v), m(u, v)$, and $b(v, q)$ as follows.

$$
\begin{aligned}
& a(u, v)=(H \nabla u, \nabla v)_{\left\{L^{2}(\Omega)\right\}^{4}}=\sum_{i, j=1}^{2} \int_{\Omega} H \frac{\partial u_{i}}{\partial x_{j}} \frac{\partial v_{i}}{\partial x_{j}} d x \\
& \text { for } u, v \in\left\{H^{1}(\Omega)\right\}^{2}, \\
& l(u, v)=(H(b, \nabla) u, v)_{\left\{L^{2}(\Omega)\right\}^{2}}=\sum_{i, j=1}^{2} \int_{\Omega} H b_{i} \frac{\partial u_{j}}{\partial x_{i}} v_{j} d x \\
& \text { for } u \in\left\{H^{1}(\Omega)\right\}^{2}, v \in\left\{L^{2}(\Omega)\right\}^{2}
\end{aligned}
$$

(2.11) $m(u, v)=(H[f] u, v)_{\left\{L^{2}(\Omega)\right\}^{2}}+g_{1}(|b| u, v)_{\left\{L^{2}(\Omega)\right\}^{2}}$ for $u, v \in\left\{L^{2}(\Omega)\right\}^{2}$,

(2.12) $b(v, q)=-g(\operatorname{div}(H v), q)_{L^{2}(\Omega)}=-g \sum_{i=1}^{2} \int_{\Omega} \frac{\partial}{\partial x_{i}}\left(H v_{i}\right) \cdot q d x$

$$
\text { for } v \in\left\{H^{1}(\Omega)\right\}^{2}, q \in L^{2}(\Omega) \text {. }
$$

Let $A$ be the selfadjoint operator acting in $X$ associated to the form $a(u, v)$ with form domain $D(a)=\left(H_{0}{ }^{1}(\Omega)\right)^{2}$. Namely, we have

(2.13) $D(A)=\left\{u \in\left(H_{0}^{1}(\Omega)\right)^{2}: \quad\right.$ There is a constant $C$ depending on $u$ such that

$$
\left.|a(u, v)| \leq C\|v\|_{X} \text { for } v \in\left(H_{0}^{1}(\Omega)\right)^{2}\right\},
$$

and

$$
A u=-\frac{1}{H}(\nabla, H \nabla) u \quad \text { for } \quad u \in D(A) .
$$

Since the boundary $\Gamma$ of $\Omega$ is $C^{\infty}$,

$$
D(A)=\left(H_{0}^{1}(\Omega) \cap H^{2}(\Omega)\right)^{2},
$$

which clarifies the meaning of (2.14). Let $L$ be the operator acting in $X$ with the domain $D(L)=\left(H^{1}(\Omega)\right)^{2}$ defined by

$$
L u=(b, \nabla) u \quad \text { for } \quad u \in D(L) .
$$

Then clearly we have

$$
l(u, v)=(L u, v)_{X} \quad \text { for } \quad u \in D(L), v \in X .
$$

Let $M$ be the bounded operator on $X$ defined by

$$
M u=[f] u+g_{1} \frac{|b|}{H} u \quad \text { for } \quad u \in X
$$

Then we have 


$$
m(u, v)=(M u, v)_{X} \quad \text { for } \quad u, v \in X .
$$

Further let $B$ be the operator with the domain $D(B)=\left\{H_{0}{ }^{1}(\Omega)\right\}^{2}$ and the range $R(B) \subset Q$, defined by

$$
B u=-\operatorname{div}(H u) \quad \text { for } \quad u \in D(B) .
$$

Then we have

$$
b(v, q)=(B v, q)_{Q} \quad \text { for } \quad v \in D(B), \quad q \in Q .
$$

Since $D(B)$ is dense in $X$, we can define the adjoint operator $B^{*}$ with domain $D\left(B^{*}\right) \subset Q$ and range $R\left(B^{*}\right) \subset X$ satisfying

$$
(B v, q)_{Q}=\left(v, B^{*} q\right)_{X} \quad \text { for } \quad v \in D(B) \text { and } q \in D\left(B^{*}\right) \text {. }
$$

In this case, it holds that

$$
D\left(B^{*}\right)=H^{1}(\Omega)
$$

and that

$$
B^{*} q=g \cdot \operatorname{grad} q \quad \text { for } \quad q \in D\left(B^{*}\right) .
$$

Finally let $T$ be the closed linear operator acting in $X$ defined by

$$
T u=v A u+L u+M u \quad \text { for } \quad u \in D(T)=D(A) .
$$

Now we can define the linear operator $\boldsymbol{A}_{0}$ acting in $\boldsymbol{X}$ through the following matrix representation:

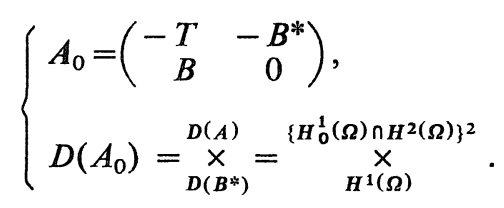

Namely for $u=\left(\begin{array}{c}u \\ p\end{array}\right) \in D\left(A_{0}\right)$,

$$
A_{0} u=\left(\begin{array}{c}
-\left(T u+B^{*} p\right) \\
B u
\end{array}\right)
$$

It is easy to see that a classical solution $u(t)=\left(\begin{array}{l}u(t, x) \\ p(t, x)\end{array}\right)$ of $(\mathscr{E})$ satisfies the following evolution equation $\left(\boldsymbol{E}_{0}\right)$ in $\boldsymbol{X}$.

$$
\frac{d u}{d t}=A_{0} u, t>0, u(0)=a \quad \text { with } \quad \boldsymbol{a}=\left(\begin{array}{c}
u^{0}(x) \\
p^{0}(x)
\end{array}\right) .
$$




\section{Proposition 2.1.}

$$
\begin{aligned}
& \left(\boldsymbol{A}_{0} \boldsymbol{u}, \boldsymbol{u}\right)_{\mathbf{X}} \leq 0 \quad \text { for } \boldsymbol{u} \in D\left(\boldsymbol{A}_{0}\right) . \\
& R\left(\lambda-\boldsymbol{A}_{0}\right) \text { is dense in } \boldsymbol{X} \text { for } \lambda>0 .
\end{aligned}
$$

Proof. (1) follows from the definition of $A_{0}$. (Note definitions from (2.6) to (2.24)).

(2) Let $\mathscr{D}(\Omega)$ be the totality of $C^{\infty}$ functions whose supports are compact in

$\Omega$. To have this assertion, it is sufficient to show the existence of $u=\left(\begin{array}{c}u \\ p\end{array}\right) \epsilon$ $D\left(\boldsymbol{A}_{0}\right)$ satisfying

$$
\left\{\begin{aligned}
&(T+\lambda) u+B^{*} p=f, \\
&-B u+\lambda p=g, \\
& \text { for } f \in\{\mathscr{D}(\Omega)\}^{2} \text { and } g \in \mathscr{D}(\Omega) .
\end{aligned}\right.
$$

If we eliminate $p$ from $\left(\boldsymbol{E}_{0}^{\lambda}\right)$ then we have, at least formally,

$$
\left(T+\lambda+\frac{1}{\lambda} B^{*} B\right) u=f-\frac{1}{\lambda} B^{*} g
$$

with

$$
p=\frac{1}{\lambda}(g+B u)
$$

Using the corresponding variational representation of the problem (2.25), we can show that the problem (2.25) has the unique solution $u \in\left\{H_{0}{ }^{1}(\Omega) \cap H^{2}(\Omega)\right\}^{2}$ taking account of the regularity property of the solution of the boundary value problem of the strongly elliptic systems (see e.g. Fichera [1]). Therefore $p$, defined by (2.26) for this $u$, belongs to $H^{1}(\Omega)$. Hence $u=\left(\begin{array}{l}u \\ p\end{array}\right) \in D\left(A_{0}\right)$ satisfies $\left(\boldsymbol{E}_{0}^{\lambda}\right)$.

Theorem 2.2. The operator $\boldsymbol{A}_{0}$ has the closure $\boldsymbol{A}$, which generates the semi-group of linear operators, $T(t)=e^{t \boldsymbol{A}}, t>0$, of class $C_{0}$ satisfying $\|\boldsymbol{T}(t) \boldsymbol{a}\|_{\boldsymbol{X}} \leq\|\boldsymbol{a}\|_{\mathbf{X}}$ for $\boldsymbol{a} \in \boldsymbol{X}$.

Proof. The assertion is a consequence of the standard theory of contraction semi-group (see e.g. Yosida [20], Krein [11]).

It is to be noted that for $\boldsymbol{a} \in D(\boldsymbol{A}), \boldsymbol{u}(t)=\boldsymbol{T}(t) \boldsymbol{a}$ is the unique solution of the evolution problem in $\boldsymbol{X}$ :

$$
\frac{d u}{d t}=A u, \quad t>0, \quad \boldsymbol{u}(0)=\boldsymbol{a} .
$$

For $\boldsymbol{a} \in \boldsymbol{X}, \boldsymbol{u}(t)=\boldsymbol{T}(t) \boldsymbol{a}$ is said to be the generalized solution of $(\boldsymbol{E})$. 


\section{§3. A Finite Element Approximation of the Model Linear System}

Let $W$ be $H^{1}(\Omega)$, and let $V$ be the totality of 2-vector functions $v=\left(v_{i}\right)_{i=1,2}$ whose components $v_{i}$ belong to $H_{0}{ }^{1}(\Omega)$. Assume that we have families of finite dimensional subspaces $\left\{V_{h}: 0<h \leq h_{\max }\right\}$ of $V$, and $\left\{Q_{h}: 0<h \leq h_{\max }\right\}$ of $W$ satisfying the following properties (3.1) to (3.3):

$$
\left\{\begin{array}{l}
\text { There are linear operators } \pi_{h} \text { from } V \cap\left(H^{2}(\Omega)\right)^{2} \text { onto } V_{h} \text {, and } \rho_{h} \text { from } \\
H^{2}(\Omega) \text { onto } Q_{h} .
\end{array}\right.
$$

$$
\left\{\begin{array}{l}
\text { There are positive constants } c_{0} \text { and } c_{1} \text { independent of } h \text { such that } \\
\left|\pi_{h} v-v\right|_{1} \leq c_{0} h|v|_{2}, \quad v \in V \cap\left(H^{2}(\Omega)\right)^{2} \\
\left|\rho_{h} w-w\right|_{0}+\left|\rho_{h} w-w\right|_{1} \leq c_{1} h|w|_{2}, \quad w \in H^{2}(\Omega) .
\end{array}\right.
$$

$$
\left\{\begin{array}{l}
\text { There are positive constants } \sigma_{0} \text { and } \sigma_{1} \text { independent of } h \text { such that } \\
\left|v_{h}\right|_{1} \leq \frac{\sigma_{0}}{h}\left|v_{h}\right|_{0}, \quad v_{h} \in V_{h}, \\
\left|w_{h}\right|_{1} \leq \frac{\sigma_{1}}{h}\left|w_{h}\right|_{0}, \quad w_{h} \in Q_{h} .
\end{array}\right.
$$

In the above and hereafter, we employ the usual semi-norm notations of Sobolev-spaces. Namely for $w \in H^{m}(\Omega), \quad|w|_{m}=\left(\sum_{i+j=m}\left|\frac{\partial^{m} w}{\partial x_{1}^{i} \partial x_{2}^{j}}\right|_{0}^{2}\right)^{1 / 2}$ with $|w|_{0}=\left(\int_{\Omega}|w|^{2} d x\right)^{1 / 2}$, and for $v=\left(v_{i}\right)_{i=1,2} \in\left(H^{m}(\Omega)\right)^{2}, \quad|v|_{m}=\left(\left|v_{1}\right|_{m}{ }^{2}+\left|v_{2}\right|_{m}^{2}\right)^{1 / 2}$. Now we admit that the following problem $\left(\Pi_{0}\right)$ is the weak formulation of $(\mathscr{E})$.

$$
\left(\Pi_{0}\right)\left\{\begin{array}{l}
\text { Find } u(t) \in V \text { and } p(t) \in Q, t \geq 0, \text { such that for } t>0, \\
\frac{d}{d t}(u(t), v)_{X}+t(u(t), v)+b(v, p(t))=0 \text { for } v \in V, \\
\frac{d}{d t}(p(t), q)_{Q}-b(u(t), q)=0 \text { for } q \in Q \\
\text { with } u(0)=u^{0} \in V \text { and } p(0)=p^{0} \in Q .
\end{array}\right.
$$

It is noted that the solution $u(t)=\{u(t), p(t)\}$ of $(E)$ satisfies $\left(\Pi_{0}\right)$ if $u(t) \in D\left(A_{0}\right)$ for any $t>0$.

As a semi-discrete Galerkin approximation of $\left(\Pi_{0}\right)$, we put the following problem $\left(\Pi_{h}\right)$.

$\left(\Pi_{h}\right)\left\{\begin{array}{l}\text { Find } u_{h}(t) \in V_{h} \text { and } p_{h}(t) \in Q_{h}, t \geq 0, \text { such that for } t>0, \\ \frac{d}{d t}\left(u_{h}(t), v_{h}\right)_{X}+t\left(u_{h}(t), v_{h}\right)+b\left(v_{h}, p_{h}(t)\right)=0 \text { for } v_{h} \in V_{h},\end{array}\right.$ 


$$
\mid \begin{aligned}
& \frac{d}{d t}\left(p_{h}(t), q_{h}\right)_{Q}-b\left(u_{h}(t), q_{h}\right)=0 \text { for } q_{h} \in Q_{h} \\
& \text { with } u_{h}(0)=u_{h}^{0} \in V_{h} \text { and } p_{h}(0)=p_{h}^{0} \in Q_{h} .
\end{aligned}
$$

As for the discretization in the time variable $t$, we adopt the following explicit scheme $\left(\Pi_{h, \tau}\right)$.

$$
\left(\Pi_{h, \tau}\right)\left\{\begin{array}{l}
\text { Find step functions } u_{h}(t) \in V_{h} \text { and } p_{h}(t) \in Q_{h} \text { with time step } \tau \text { such that } \\
\text { for } t \in[n \tau,(n+1) \tau), n=0,1,2, \ldots, \\
\left(\left(D_{\tau} u_{h}\right)(t), v_{h}\right)_{X}+t\left(u_{h}(t), v_{h}\right)+b\left(v_{h}, p_{h}(t)\right)=0 \text { for } v_{h} \in V_{h}, \\
\left(\left(D_{\tau} p_{h}\right)(t), q_{h}\right)_{Q}-b\left(u_{h}(t+\tau), q_{h}\right)=0 \text { for } q_{h} \in Q_{h} \\
\text { with } u_{h}(t)=u_{h}^{0} \in V_{h} \text { and } p_{h}(t)=p_{h}^{0} \in Q_{h} \text { for } t \in[0, \tau)
\end{array}\right.
$$

Here we used the notation $\left(D_{\tau} u\right)(t)=-\frac{u(t+\tau)-u(t)}{\tau}$.

From Poincaré-Friedrichs inequality, there is a constant $\alpha_{0}$ such that

$$
|v|_{1} \geq \alpha_{0}|v|_{0} \quad \text { for } \quad v \in V .
$$

Using (3.3) and (3.4), we can conclude that there are positive constants $\alpha, \sigma, \gamma$ such that

$$
\begin{aligned}
& a(v, v)^{1 / 2} \geq \alpha\|v\|_{X} \text { for } v \in V, \\
& a\left(v_{h}, v_{h}\right)^{1 / 2} \leq \frac{\sigma}{h}\left\|v_{h}\right\|_{X} \text { for } v_{h} \in V_{h}, \\
& \|\operatorname{div}(H v)\|_{Q} \leq \gamma a(v, v)^{1 / 2} \text { for } v \in V .
\end{aligned}
$$

In fact, we can take

$$
\alpha=\alpha_{0}(\underline{H} / \bar{H})^{1 / 2}, \sigma=\sigma_{0}(\bar{H} / \underline{H})^{1 / 2},
$$

by (2.3) and

$$
\gamma=(g \bar{H})^{1 / 2}\left(1+\frac{1}{\alpha_{0}} \sup _{x \in \Omega}|\nabla H(x)|_{R^{2}}\right)
$$

by (2.1) and (2.3).

The following two theorems are main results of this paper.

Theorem 3.1. Let $\left\{u_{h}(t), p_{h}(t)\right\}$ be a solution of $\left(\Pi_{h}\right)$, and let $u(t)=$ $\{u(t), p(t)\}$ be a generalized solution of $(E)$. If $\lim _{h \rightarrow 0}\left(\left\|u_{h}^{0}-u^{0}\right\|_{X}+\| p_{h}^{0}\right.$ $\left.-p^{0} \|_{Q}\right)=0$, then we have $\lim _{h \vdash 0}\left(\left\|u_{h}(t)-u(t)\right\|_{X}+\left\|p_{h}(t)-p(t)\right\|_{Q}\right)=0$ uniformly in $t \in[0, T]$ for any finite $T$.

Theorem 3.2. Let $\left\{u_{h}(t), p_{h}(t)\right\}$ be a solution of $\left(\Pi_{h, \tau}\right)$, and let $u(t)=$ 
$\{u(t), p(t)\}$ be a generalized solution of $(\mathbb{E})$. If $\lim _{h \rightarrow 0}\left(\left\|u_{h}^{0}-u^{0}\right\|_{X}+\left\|p_{h}^{0}-p^{0}\right\|_{Q}\right)$ $=0$, then we have $\lim _{h \downarrow 0}\left(\left\|u_{h}(t)-u(t)\right\|_{X}+\left\|p_{h}(t)-p(t)\right\|_{Q}\right)=0$ uniformly in $t \in$ $[0, T]$ for any finite $T$ provided that $\tau$ in $\left(\Pi_{h, \tau}\right)$ is chosen so as to satisfy

$$
\tau \leq 2 v \min \left\{\frac{\varepsilon}{(v \sigma+\beta h)^{2}} h^{2}, \frac{\delta}{\gamma^{2}}\right\}
$$

with arbitrary positive $\varepsilon$ and $\delta$ such that $\varepsilon+\delta \leq 1$, where

$$
\left\{\begin{array}{l}
\beta=\beta_{0}+m / \alpha, \\
\beta_{0}=\sup _{x \in \Omega} \max _{i=1,2}\left|b_{i}(x)\right|, \\
m=|f|+g_{1} \beta_{0} / \underline{H} .
\end{array}\right.
$$

Solvability of $\left(\Pi_{h}\right)$ is obvious since it is essentially a system of 1st order ordinary differential equations with constant coefficient which can be transformed to be of normal form.

As an example of finite element spaces which satisfy (3.1) to (3.3), we quote here piecewise linear continuous finite element spaces with Zlamal's curved element technique (see [21]). Let $\mathscr{T}_{h}$ be a "triangulation" of the domain $\Omega$. Here $h$ is the representative length of the "triangulation". Curved elements duc to Zlamal are adopted near the boundary $\Gamma$, while the simplest triangular elements are used in the interior of $\Omega$. It is assumed that there is given a regular famliy of "triangulation" of $\Omega,\left\{\mathscr{T}_{h}: 0<h \leq h_{\max }<\infty\right\}$, with the inverse assumption. Namely, there are two positive constants $\Sigma$ and $\Lambda$ such that $h_{T} / \rho_{T} \leq \Sigma$ and $h / h_{T} \leq \Lambda$, where $h_{T}$ and $\rho_{T}$ denote the diameter of a "triangular" element $T$ (the usual one or the curved one) and the maximum of diameters of inscribed circles of $T$, respectively. Let $W_{h}$ be the "piecewise-linear" continuous finite element space constructed by $\mathscr{T}_{h}: W_{h}=\left\{w_{h} \in C(\bar{\Omega}): w_{h}\right.$ is a "polynomial" with degree at most 1 on each "triangle" of $\left.\mathscr{T}_{h}\right\}$. A little more precisely, $w_{h} \in W_{h}$ is a polynomial with degree at most 1 on each triangle element of $\mathscr{T}_{h}$, while on a curved element $T, w_{h}\left(F_{T}(\hat{x})\right)$ is a polynomial with degree at most 1 on a reference triangle $\hat{T}$, where $F_{T}: \hat{T} \ni \hat{x} \rightarrow x=F_{T}(\hat{x}) \in T$ is the isomorphism from $\hat{T}$ onto $T$ determined by Zlamal's method.

Let $Q_{h}=W_{h}$, and let

$$
V_{h}=\left\{v_{h} \in\left(W_{h}\right)^{2}:\left.v_{h}\right|_{\Gamma}=0\right\} .
$$

Then $V_{h}$ and $Q_{h}$ satisfy conditions (3.1), (3.2) and (3.3). 


\section{§4. Proofs of Theorems 3.1 and 3.2}

In this section, the space $V_{h}$ is considered to be the Hilbert space $X_{h}$ with the inner product induced from the space $X$. Also the space $Q_{h}$ is the Hilbert space with the inner product induced from $Q$. Let $\boldsymbol{X}_{\boldsymbol{h}}$ be the product Hilbert space of $X_{h}$ and $Q_{h}$ :

$$
X_{h}=\stackrel{X_{h}}{\stackrel{\times}{Q_{h}}}
$$

Let $P_{h}$, and $R_{h}$, be orthogonal projections from $X$, and $Q$, onto $X_{h}$, and $Q_{h}$, respectively. Let $\boldsymbol{P}_{h}$ be the orthogonal projection from $\boldsymbol{X}$ onto $\boldsymbol{X}_{h}$. Using Aubin-Nitsche duality technique, we can deduce from conditions (3.1) to (3.3) that there are positive constants $C_{0}$ and $C_{1}$ such that

$$
\begin{array}{ll}
\left\|P_{h} v-v\right\|_{X} \leq C_{0} h\|v\|_{H^{1}(\Omega)} & \text { for } \quad v \in V, \\
\left\|R_{h} q-q\right\|_{Q} \leq C_{1} h\|q\|_{H^{1}(\Omega)} & \text { for } \quad q \in W .
\end{array}
$$

By (4.1) and (4.2), we have that $\boldsymbol{X}_{h} \stackrel{K}{\longrightarrow} \boldsymbol{X}$ with the approximating operator $\boldsymbol{P}_{h}$ as $h$ tends to 0 .

Now we can define bounded linear operators $A_{h}, L_{h}, M_{h} \in L\left(X_{h}\right)$, and $B_{h} \in L\left(X_{h}, Q_{h}\right)$ so as to satisfy the following relations:

$$
\begin{aligned}
\left(A_{h} u_{h}, v_{h}\right)_{X_{h}}= & a\left(u_{h}, v_{h}\right), \\
\left(L_{h} u_{h}, v_{h}\right)_{X_{h}}= & l\left(u_{h}, v_{h}\right), \\
\left(M_{h} u_{h}, v_{h}\right)_{X_{h}}= & m\left(u_{h}, v_{h}\right), \\
\left(B_{h} v_{h}, q_{h}\right)_{Q_{h}}= & b\left(v_{h}, q_{h}\right), \\
& \text { for any } u_{h}, v_{h} \in X_{h} \text { and } q_{h} \in Q_{h} .
\end{aligned}
$$

Hence we can define

$$
T_{h}=v A_{h}+L_{h}+M_{h} \in L\left(X_{h}\right)
$$

and

$$
A_{h}=\left(\begin{array}{rc}
-T_{h} & -B_{h}^{*} \\
B_{h} & 0
\end{array}\right) \in L\left(X_{h}\right) .
$$

Then the problem $\left(\Pi_{h}\right)$ is transformed to the following operational form $\left(E_{h}\right)$. 
$\left(E_{h}\right)$

$$
\left\{\begin{array}{l}
\frac{d}{d t} \boldsymbol{u}_{h}(t)=\boldsymbol{A}_{h} \boldsymbol{u}_{h}(t), \quad t>0, \\
\boldsymbol{u}_{h}(0)=\boldsymbol{u}_{h}^{0}
\end{array}\right.
$$

Here we use the notation $\boldsymbol{u}_{h}(t)=\left(\begin{array}{c}u_{h}(t) \\ p_{h}(t)\end{array}\right)$, which is considered as an $\boldsymbol{X}_{h}$-valued function.

\section{Proposition 4.1.}

$$
\begin{aligned}
& \left(A_{h} v_{h}, v_{h}\right)_{X_{h}} \leq 0 \quad \text { for } \quad v_{h} \in X_{h} \text {, } \\
& R\left(\lambda-A_{h}\right)=X_{h} \quad \text { for } \quad \lambda>0 \text {. }
\end{aligned}
$$

Proof. (1) comes from the definition of operator $\boldsymbol{A}_{h}$. (Note definitions from (4.3) to (4.8).) Hence we have

$$
\left(\left(\lambda-A_{h}\right) v_{h}, v_{h}\right)_{X_{h}} \geq \lambda\left\|v_{h}\right\|_{X_{h}}^{2} .
$$

Therefore $N\left(\lambda-\boldsymbol{A}_{h}\right)=\{0\}$. The finite dimensionality of $\boldsymbol{X}_{h}$ implies (2).

Now we consider the following resolvent equations $\left(E^{\lambda}\right)$ and $\left(E_{h}^{\lambda}\right)$ for $\lambda>0$.

$$
(A-\lambda) \boldsymbol{u}=\boldsymbol{f} .
$$

$$
\left(A_{h}-\lambda\right) \boldsymbol{u}_{h}=\boldsymbol{f}_{h} \text {. }
$$

Proposition 4.2. If $\boldsymbol{f} \in\{\mathscr{D}(\bar{\Omega})\}^{3}$, for $\boldsymbol{f}_{h}=\boldsymbol{P}_{h} \boldsymbol{f}$ we have

$$
\left\|\boldsymbol{u}_{h}-\boldsymbol{u}\right\|_{\mathbf{x}}=O(h) \text {. }
$$

Proof. See Kanayama-Ushijima [7].

From Proposition 4.2, we have

$$
\left(A_{h}-\lambda\right)^{-1} \stackrel{K}{\longrightarrow}(A-\lambda)^{-1} .
$$

From (1) of Proposition 4.1, it holds

$$
\left\|e^{t A_{h}} a_{h}\right\|_{\boldsymbol{x}_{h}} \leq\left\|\boldsymbol{a}_{h}\right\|_{\boldsymbol{X}_{h}}, \quad \boldsymbol{a}_{h} \in \boldsymbol{X}_{h} .
$$

By Theorem 1.1, (4.A) and (4.B) imply

$$
\text { (4.C) } \quad e^{t A_{h} \stackrel{K}{\longrightarrow}} T(t) \text { uniformly in } t \in[0, T] \text { for any finite } T \text {. }
$$

By a routine argument, we have Theorem 3.1.

Now we proceed to the problem $\left(\Pi_{h, \tau}\right)$, which is represented in the following operational form $\left(E_{\tau}\right)$ : 
$\left(E_{\tau}\right) \quad\left\{\begin{array}{l}\frac{u_{n}-u_{m}}{\tau}+T u_{m}+B^{*} p_{m}=0, \\ \frac{p_{n}-p_{m}}{\tau}-B u_{n}=0, \quad \text { for } n=m+1, m=0,1,2, \ldots \\ u_{0}=u^{0}, p_{0}=p^{0},\end{array}\right.$

where, for simplicity of notations, we drop the suffix $h$. Namely we should understand in $\left(E_{\mathrm{\tau}}\right)$

$$
\left\{\begin{array}{l}
u_{n}=u_{h}(n \tau), \quad u_{m}=u_{h}(m \tau), \\
p_{n}=p_{h}(n \tau), \quad p_{m}=p_{h}(m \tau), \\
T=T_{h}, \quad B=B_{h},
\end{array}\right.
$$

where right hand sides of (4.9) are the vectors or operators appeared in $\left(\Pi_{h, \tau}\right)$. $\left(E_{\tau}\right)$ is also rewritten in the following form $\left(\boldsymbol{E}_{\tau}\right)$

( $\left.\boldsymbol{E}_{\tau}\right)\left\{\begin{array}{l}\frac{\boldsymbol{u}_{n}-\boldsymbol{u}_{m}}{\tau}=\boldsymbol{A}_{\tau} \boldsymbol{u}_{m}, \quad n=m+1, m=0,1, \ldots, \\ \boldsymbol{u}_{0}=\boldsymbol{u}^{0},\end{array}\right.$

where $\boldsymbol{u}_{n}=\left(\begin{array}{c}u_{n} \\ p_{n}\end{array}\right)$ and

$$
A_{\mathrm{\tau}}=A+\tau B, \quad \text { with } \quad \boldsymbol{B}=\left(\begin{array}{cc}
0 & 0 \\
-B T & -B B^{*}
\end{array}\right) .
$$

As for the stability of the scheme $\left(E_{\tau}\right)$, we have the following.

Proposition 4.3. Consider the recurrence formula $\left(E_{\tau}\right)$ in Hilbert spaces $X$ and $Q$. Let $T \in L(X), B \in L(X, Q)$ in $\left(E_{\tau}\right)$. Let $|v|_{T}=(T v, v)^{1 / 2}$. Assume that there are positive constants $a$ and $b$ such that

$$
\left\{\begin{array}{l}
(T u, v)_{X} \leq a\|u\|_{X}|v|_{T}, \\
(B v, q)_{Q} \leq b|v|_{T}\|q\|_{Q} .
\end{array}\right.
$$

Then we have in the scheme $\left(E_{\tau}\right)$,

$$
\left\|u_{n}\right\|_{X}^{2}+\left\|p_{n}\right\|_{Q}^{2} \leq\left\|u_{0}\right\|_{X}{ }^{2}+\left\|p_{0}\right\|_{Q}^{2} \quad n=1,2,3, \ldots,
$$

provided that $\tau$ is chosen so as 10 satisfy

$$
1-\frac{a^{2}}{2 \varepsilon} \tau \geq 0 \text { and } 1-\frac{b^{2}}{2 \delta} \tau \geq 0
$$

with arbitrary positive constants 5 and $\delta$ such that

$$
1-\varepsilon-\delta \geq 0 \text {. }
$$


Proof. From the first equality of $\left(E_{\tau}\right)$, we have

$$
\left(\frac{u_{n}-u_{m}}{\tau}, u_{n}\right)_{X}+\left(T u_{m}, u_{n}\right)_{X}+\left(u_{n}, B^{*} p_{m}\right)_{X}=0 .
$$

From the second, we get

$$
\left(\frac{p_{n}-p_{m}}{\tau}, p_{n}\right)_{Q}-\left(B u_{n}, p_{n}\right)_{Q}=0
$$

Adding (4.15) and (4.16), we obtain

$$
\left(\frac{u_{n}-u_{m}}{\tau}, u_{n}\right)_{X}+\left(\frac{p_{n}-p_{m}}{\tau}, p_{n}\right)_{Q}+\left(T u_{m}, u_{n}\right)_{X}+\left(B u_{n}, p_{m}-p_{n}\right)_{Q}=0
$$

Using the identity: $(a-b, a)=\frac{1}{2}\left\{\|a\|^{2}-\|b\|^{2}+\|a-b\|^{2}\right\},(4.17)$ is rewritten

$$
\left\{\begin{array}{l}
\quad \frac{1}{2 \tau}\left\{\left\|u_{n}\right\|_{X}^{2}-\left\|u_{m}\right\|_{X}^{2}+\left\|u_{n}-u_{m}\right\|_{X}{ }^{2}\right\} \\
+\frac{1}{2 \tau}\left\{\left\|p_{n}\right\|_{Q}^{2}-\left\|p_{m}\right\|_{Q}^{2}+\left\|p_{n}-p_{m}\right\|_{Q^{2}}\right\} \\
+\left(T u_{n}, u_{n}\right)_{X} \\
=\left(T\left(u_{n}-u_{m}\right), u_{n}\right)_{X}+\left(B u_{n}, p_{n}-p_{m}\right)_{Q} .
\end{array}\right.
$$

Then (4.11) implies

the right hand side of (4.18)

$$
\begin{aligned}
& \leq a\left\|u_{n}-u_{m}\right\|_{X}\left|u_{n}\right|_{T}+b\left\|p_{n}-p_{m}\right\|_{Q}\left|u_{n}\right|_{T} \\
& \leq \varepsilon\left|u_{n}\right|_{T}^{2}+\frac{1}{\varepsilon} \frac{a^{2}}{4}\left\|u_{n}-u_{m}\right\|_{X}{ }^{2}+\delta\left|u_{n}\right|_{T}{ }^{2}+\frac{1}{\delta} \frac{b^{2}}{4}\left\|p_{n}-p_{m}\right\|_{Q}{ }^{2} .
\end{aligned}
$$

Combining this estimate with (4.18), we have

$$
\left\{\begin{array}{l}
\frac{1}{2} D_{\tau}\left\{\left\|u_{m}\right\|_{X}{ }^{2}+\left\|p_{m}\right\|_{Q^{2}}\right\}+\frac{1}{2 \tau}\left\{1-\frac{a^{2}}{2 \varepsilon} \tau\right\}\left\|u_{n}-u_{m}\right\|_{X}{ }^{2} \\
+\frac{1}{2 \tau}\left\{1-\frac{b^{2}}{2 \delta} \tau\right\}\left\|p_{n}-p_{m}\right\|_{Q^{2}}+(1-\varepsilon-\delta)\left|u_{n}\right|_{T}^{2} \leq 0
\end{array}\right.
$$

Condition (4.13) and estimate (4.19) imply

$$
\left\|u_{n}\right\|_{X}^{2}+\left\|p_{n}\right\|_{Q^{2}} \leq\left\|u_{m}\right\|_{X}{ }^{2}+\left\|p_{m}\right\|_{Q^{2}}
$$

Hence (4.12) holds.

Remark. The essence of the above proof comes from the criterion for Arrow-Hurwicz algorithm given in Temam [15].

Proposition 4.4. The solution $u_{h}(t)=\left\{u_{h}(t), p_{h}(t)\right\}$ of the scheme $\left(\Pi_{h, \tau}\right)$ 
satisfies

$$
\left\|\boldsymbol{u}_{h}(t)\right\|_{\mathbf{x}_{h}} \leq\left\|\boldsymbol{u}_{h}(0)\right\|_{\mathbf{x}_{h}} \quad \text { for any } \quad t \geq 0
$$

provided that $\tau$ is chosen so as to satisfy' the condition (3.10).

Proof. Firstly we decompose $m(u, v)=m_{0}(u, v)+m_{1}(u, v)$ as

$$
\left\{\begin{array}{l}
m_{0}(u, v)=(H[f] u, v)_{\left\{L^{2}(\Omega)\right\}^{2}}, \\
m_{1}(u, v)=g_{1}(|b| u, v)_{\left\{L^{2}(\Omega)\right\}^{2}} .
\end{array}\right.
$$

Noticing that the definition of $[f]$ implies

$$
m_{0}(u, v)=-m_{0}(v, u) \quad \text { for } \quad u, v \in X,
$$

and that (2.2) and (2.4) imply

$$
l(u, v)=-l(v, u) \quad \text { for } \quad u, v \in V,
$$

we have

$$
t(v, v)=v a(v, v)+m_{1}(v, v) \quad \text { for } \quad v \in V .
$$

Hence it holds

$$
t(v, v) \geq v a(v, v) \quad \text { for } \quad v \in V .
$$

From (4.23), we obtain

$$
t(u, v)=v a(u, v)-l(v, u)+m(u, v) \quad \text { for } \quad u, v \in V .
$$

Now we take $u_{h}$ and $v_{h}$ being elements of $X_{h}=V_{h}$. Then

$$
\begin{aligned}
v a\left(u_{h}, v_{h}\right) & \leq\left(v a\left(u_{h}, u_{h}\right)\right)^{1 / 2}\left(v a\left(v_{h}, v_{h}\right)\right)^{1 / 2} \\
& \leq \frac{\sqrt{v} \sigma^{1 / 2}}{h}\left\|u_{h}\right\|_{X}\left(t\left(v_{h}, v_{h}\right)\right)^{1 / 2} \\
& \text { by (3.6) and (4.25). }
\end{aligned}
$$

Namely, we have

$$
v a\left(u_{h}, v_{h}\right) \leq \frac{\sqrt{v \sigma}}{h}\left\|u_{h}\right\|_{X_{h}}\left|v_{h}\right|_{T_{h}}
$$

where $\left|v_{h}\right|_{T_{h}}=\left(T_{h} v_{h}, v_{h}\right)_{X_{h}}{ }^{1 / 2}$. Since $l\left(v_{h}, u_{h}\right)=\left(H(b, \nabla) v_{h}, u_{h}\right)$, letting $\beta_{0}=$ $\sup _{x \in \Omega} \max _{i=1,2}\left|b_{i}(x)\right|$, we have

$$
-l\left(v_{h}, u_{h}\right) \leq \beta_{0}\left\|u_{h}\right\|_{X_{h}} a\left(v_{h}, v_{h}\right)^{1 / 2} .
$$

Therefore by (4.25) it holds

$$
-l\left(v_{h}, u_{h}\right) \leq \frac{\beta_{0}}{\sqrt{v}}\left\|u_{h}\right\|_{X_{h}}\left|v_{h}\right|_{T_{h}}
$$


It is easy to see that

$$
m(u, v) \leq m\|u\|_{X}\|v\|_{X} \quad \text { for } \quad u, v \in X,
$$

with

$$
m=|f|+g_{1} \beta_{0} / \underline{H} .
$$

Combining with (4.29), (3.5), and (4.25) we have

$$
m\left(u_{h}, v_{h}\right) \leq \frac{m}{\sqrt{v \alpha}}\left\|u_{h}\right\|_{X_{h}}\left|v_{h}\right|_{T_{h}} .
$$

From the euqality (4.26) and estimates (4.27), (4.28) and (4.31), the following estimate (4.32) is ohtained.

$$
\left(T_{h} u_{h}, v_{h}\right)_{X_{h}} \leq \frac{v \sigma+\beta h}{\sqrt{v h}}\left\|u_{h}\right\|_{X_{h}}\left|v_{h}\right|_{T_{h}} \quad \text { for } \quad u_{h}, v_{h} \in X_{h}
$$

with

$$
\beta=\beta_{0}+m / \alpha .
$$

Analogously, for $v_{h} \in X_{h}$ and $q_{h} \in Q_{h}$, we have

$$
\begin{array}{rlr}
\left(B_{h} v_{h}, q_{h}\right)_{Q_{h}} & =-\left(\operatorname{div}\left(H v_{h}\right), q_{h}\right)_{Q} \\
& \leq\left\|\operatorname{div}\left(H v_{h}\right)\right\|_{Q}\left\|q_{h}\right\|_{Q_{h}} \\
& \leq \gamma a\left(v_{h}, v_{h}\right)^{1 / 2}\left\|q_{h}\right\|_{Q_{h}} \quad(\text { by }(3.7)) \\
& \leq \frac{\gamma}{\sqrt{v}} t\left(v_{h}, v_{h}\right)^{1 / 2}\left\|q_{h}\right\|_{Q_{h}} . \quad(\text { by (4.25)) }
\end{array}
$$

Thus the following estimate (4.34) is obtained.

$$
\left(B_{h} v_{h}, q_{h}\right)_{Q_{h}} \leq \frac{\gamma}{\sqrt{v}}\left|v_{h}\right|_{T_{h}}\left\|q_{h}\right\|_{Q_{h}} \quad \text { for } \quad v_{h} \in X_{h} \quad \text { and } \quad q_{h} \in Q_{h} .
$$

From (4.32) and (4.34), we can apply Proposition 4.3 to the problem $\left(\Pi_{h, \tau}\right)$ with parameters

$$
(X, Q, T, B, a, b)=\left(X_{h}, Q_{h}, T_{h}, B_{h}, \frac{v \sigma+\beta h}{\sqrt{v} h}, \frac{\gamma}{\sqrt{v}}\right) .
$$

Hence we have for the solution $\left\{u_{h}(n \tau), p_{h}(n \tau)\right\}_{n=0,1}, \cdots$,

$$
\left\|u_{h}(n \tau)\right\|_{X_{h}}{ }^{2}+\left\|p_{h}(n \tau)\right\|_{Q_{h}}{ }^{2} \leq\left\|u_{h}(0)\right\|_{X_{h}}{ }^{2}+\left\|p_{h}(0)\right\|_{Q_{h}}{ }^{2}
$$

under the condition (3.10). This estimate is the same thing as (4.20). The relation (3.11) comes from (4.30) and (4.33).

Now we proceed to consider the resolvent equation $\left(\boldsymbol{E}_{h \tau}^{\lambda}\right)$ for $\lambda>0$. 
$\left(\boldsymbol{E}_{h, \tau}^{\lambda}\right)$

$$
\left(A_{h, \tau}-\lambda\right) \boldsymbol{u}_{h, \tau}=\boldsymbol{f}_{h}
$$

with the operator

$$
A_{h, \tau}=A_{h}+\tau B_{h}, B_{h}=\left(\begin{array}{cc}
0 & 0 \\
-B_{h} T_{h} & -B_{h} B_{h}^{*}
\end{array}\right),
$$

which generates a discrete semi-group

$$
T_{h}(t)=\left(1+\tau A_{h, \tau}\right)^{[t / \tau]} \quad \text { for } \quad t \geq 0
$$

with time unit $\tau$ in $\boldsymbol{X}_{h}$. Then the solution $\boldsymbol{u}_{h}(t)$ of $\left(\Pi_{h, \tau}\right)$ satisfies $\boldsymbol{u}_{h}(t)=\boldsymbol{T}_{h}(t) \boldsymbol{a}_{h}$ for $t \geq 0$ with $\boldsymbol{a}_{h}=\boldsymbol{u}_{h}(0)$. Proposition 4.4 implies

$$
\left\|\boldsymbol{T}_{h}(t) \boldsymbol{a}_{h}\right\|_{\boldsymbol{x}_{h}} \leq\left\|\boldsymbol{a}_{h}\right\|_{\mathbf{x}_{h}} \cdot \quad a_{h} \in \boldsymbol{X}_{h}, t \geq 0,
$$

under the condition (3.10). By Proposition 3.3 of [17], (4. $\left.B_{\tau}\right)$ implies that

$$
\left\|\boldsymbol{e}^{\boldsymbol{t} \boldsymbol{A}_{h, \tau}}\right\|_{\mathrm{L}\left(\mathbf{X}_{h}\right)} \leq 1 \quad \text { for } \quad 1 \geq 0 .
$$

Hence we have that for $\lambda>0,\left(\lambda-A_{h, \tau}\right)^{-1}$ exists and satisfies

$$
\left\|\left(\lambda-A_{h, \tau}\right)^{-1}\right\|_{L\left(\mathbf{X}_{h}\right)} \leq \lambda^{-1} .
$$

Proposition 4.5 Assume that the condition (3.10) holds. Let $\boldsymbol{u}_{h . \tau}$, and $\boldsymbol{u}_{h}$, be the solutions of $\left(\boldsymbol{E}_{h, \tau}^{\lambda}\right)$, and $\left(\boldsymbol{E}_{h}^{\lambda}\right)$, respectively. Then we have

$$
\left\|\boldsymbol{u}_{h, \tau}-\boldsymbol{u}_{h}\right\|_{\boldsymbol{X}_{h}} \leq \tau\left\|B_{h}\right\|_{L\left(X_{h}, Q_{h}\right)} \frac{2\left\|\boldsymbol{f}_{h}\right\|_{\mathbf{X}_{h}}}{i} .
$$

Proof. Again, for simplicity of notations, we drop the suffix $h$ in this proof. Let $\boldsymbol{f}=\left(\begin{array}{l}f \\ g\end{array}\right), \boldsymbol{u}_{\tau}=\left(\begin{array}{l}u_{\tau} \\ p_{\tau}\end{array}\right)$ and $\boldsymbol{u}=\left(\begin{array}{l}u \\ p\end{array}\right)$. Then $\left(\boldsymbol{E}_{\tau}^{\lambda}\right)$ and $\left(\boldsymbol{E}^{\lambda}\right)$ can be represented as the following (4.38) and (4.39).

$$
\begin{aligned}
& \left\{\begin{array}{l}
(\lambda+T) u_{\tau}+B^{*} p_{\tau}=f, \\
(-B+\tau B T) u_{\tau}+\left(\lambda+\tau B B^{*}\right) p_{\tau}=g .
\end{array}\right. \\
& \left\{\begin{array}{l}
(\lambda+T) u+B^{*} p=f, \\
-B u+\lambda p=g .
\end{array}\right.
\end{aligned}
$$

Let $v_{\tau}=u_{\tau}-u$ and $q_{\tau}=p_{\tau}-p$, together with $v_{\tau}=\left(\begin{array}{c}v_{\tau} \\ g_{\tau}\end{array}\right)$. Then from (4.38) and (4.39), we have

$$
\left\{\begin{array}{l}
(\lambda+T) v_{\tau}+B^{*} q_{\tau}=0 \\
(-B+\tau B T) v_{\tau}+\left(\lambda+B B^{*}\right) p_{\tau}=\tau B(f-\lambda u) .
\end{array}\right.
$$

From (4.36) and (4.40), it holds

$$
\left\|\boldsymbol{v}_{\tau}\right\|_{\boldsymbol{X}} \leq \frac{1}{\lambda}\left\|\left(\begin{array}{c}
0 \\
\tau B(f-\lambda u)
\end{array}\right)\right\|_{\mathbf{X}} \leq \lambda^{-1} \tau\|B\|_{L(X, Q)}\|f-\lambda u\|_{X} .
$$


Since we have

$$
\begin{aligned}
\|f-\lambda u\|_{X} & \leq\|f\|_{X}+\lambda\|u\|_{X} \\
& \leq\|f\|_{X}+\lambda\|u\|_{X} \\
& =\|f\|_{X}+\lambda\left\|(\lambda-A)^{-1} f\right\|_{X} \\
& \leq\|f\|_{X}+\|f\|_{X} \quad(\text { by the dissipativity of } A) \\
& \leq 2\|f\|_{X},
\end{aligned}
$$

it holds

$$
\left\|\boldsymbol{v}_{\tau}\right\|_{\mathbf{X}} \leq \tau\|B\|_{L(X, Q)} \frac{2\|\boldsymbol{f}\|_{\mathbf{X}}}{\lambda}
$$

Thus (4.37) is proved.

Combining (3.6) and (3.7), we get

$$
\left\|B_{h}\right\|_{L\left(X_{h}, Q_{h}\right)} \leq \frac{\gamma \sigma}{h} .
$$

Hence under the condition (3.10)

$$
\tau\left\|B_{h}\right\|_{L\left(X_{h}, Q_{h}\right)} \leq \frac{2 v \varepsilon \gamma \sigma}{(v \sigma+\beta h)^{2}} h
$$

holds, which implies

$$
\left\|\left(\lambda-A_{h, \tau}\right)^{-1}-\left(\lambda-A_{h}\right)^{-1}\right\|_{L\left(\mathbf{X}_{h}\right)}=O(h) \text { as } h \rightarrow 0
$$

by (4.37). Combining this with (4.A), it is easy to see

$$
\left(\lambda-A_{h, \tau}\right)^{-1} \stackrel{K}{\longrightarrow}(\lambda-A)^{-1}
$$

under the condition (3.10). Applying Theorem 1.2 to this case, we have that $\left(4 . A_{\tau}\right)$ and $\left(4 \cdot B_{\tau}\right)$ imply

(4.C $\left.C_{\tau}\right) \quad T_{h}(t) \stackrel{K}{\longrightarrow} T(t)$ uniformly in $t \in[0, T]$ for any finite $T$,

provided that the condition (3.10) is satisfied. From $\left(4 . C_{\tau}\right)$, we get Theorem 3.2.

\section{§5. An Error Estimate in the Case of a Smooth Classical Solution}

Now we consider the case that there is a sufficiently smooth solution $\{u(t, x), p(t, x)\}$ of the problem $(\mathscr{E})$ for a specified initial value $\left\{u_{0}(x), p_{0}(x)\right\}$. Our requirements are the following. 


$$
\left\{\begin{array}{l}
\frac{\partial}{\partial t} u(t, x), \text { and } \frac{\partial^{2}}{\partial t^{2}} u(t, x), \text { can be regarded as }\left\{L^{2}(\Omega)\right\}^{2} \text {-valued con- } \\
\text { tinuous functions, } \frac{d}{d t} u(t), \text { and } \frac{d^{2}}{d t^{2}} u(t), \text { respectively, for } t \geq 0
\end{array}\right.
$$

(5.2) $\left\{u(t, x)\right.$ can be regarded as an $\left\{H^{2}(\Omega)\right\}^{2}$-valued continuous function $u(t)$ for $t \geq 0$.

$$
\left\{\begin{array}{l}
\frac{\partial}{\partial t} p(t, x), \text { and } \frac{\partial^{2}}{\partial t^{2}} p(t, x), \text { can be regarded as } L^{2}(\Omega) \text {-valued continuous } \\
\text { functions, } \frac{d}{d t} p(t), \text { and } \frac{d^{2}}{d t^{2}} p(t), \text { respectively, for } t \geq 0
\end{array}\right.
$$

$$
\left\{\begin{array}{l}
p(t, x) \text { can be regarded as an } H^{1}(\Omega) \text {-valued continuous function } p(t) \\
\text { for } t \geq 0 .
\end{array}\right.
$$

Then we have the following error estimate.

Theorem 5.1. Let the problem $(\mathscr{E})$ has the solution $\{u(t), p(t)\}$ satisfying requirements (5.1) to (5.4). Assume $\tau$ in the problem $\left(\Pi_{h, \tau}\right)$ to be chosen so as to satisfy the condition (3.10). Let $\left\{u_{h}(t), p_{h}(t)\right\}$ be the solution of $\left(\Pi_{h, \tau}\right)$ with the initial data $\left\{u_{h}^{0}, p_{h}^{0}\right\}=\left\{P_{h} u_{0}, R_{h} p_{0}\right\}$, where $P_{h}$ and $R_{h}$ are defined in Section 4. Then there is a constant $C$ depending on the positive number $T$ such that

$$
\begin{gathered}
\left\|u_{h}(t)-u(t)\right\|_{\left\{L^{2}(\Omega)\right\}^{2}}+\left\|p_{h}(t)-p(t)\right\|_{L^{2}(\Omega)} \leq C(h+\tau), \\
0 \leq t \leq T .
\end{gathered}
$$

Proof. Let $\Pi_{h}$ be the Ritz projection with respect to the bilinear form $a(u, v)$ from $V$ onto $V_{h}$. Namely $\tilde{u}_{h}=\Pi_{h} u$ for $u \in V$ is defined by the problem:

$$
\left\{\begin{array}{l}
a\left(\tilde{u}_{h}, v_{h}\right)=a\left(u, v_{h}\right) \quad \text { for } \quad v_{h} \in V_{h}, \\
\tilde{u}_{h} \in V_{h} .
\end{array}\right.
$$

Define the step function $\tilde{u}_{h}(t)$ and $\tilde{p}_{h}(t)$ by

$$
\left\{\begin{array}{l}
\tilde{u}_{h}(t)=\left(\Pi_{h} u\right)(m \tau) \\
\tilde{p}_{h}(t)=\left(R_{h} p\right)(m \tau), \\
m \tau \leq t<(m+1) \tau, \quad m=0,1,2, \ldots
\end{array}\right.
$$

Since it holds for $t \geq 0$

$$
\left\{\begin{array}{l}
\left(\frac{d u}{d t}, v\right)_{X}+v a(u, v)+l(u, v)+m(u, v)+b(v, p)=0, \quad v \in V \\
\left(\frac{d p}{d t}, q\right)_{Q}-b(u, q)=0, \quad q \in Q
\end{array}\right.
$$


(5.6) and (5.7) imply that at $t=m \tau, m=0,1,2, \ldots$,

$$
\left\{\begin{array}{l}
\left(\frac{d u}{d t}, v_{h}\right)_{X}+v a\left(\tilde{u}_{h}, v_{h}\right)+l\left(u, v_{h}\right)+m\left(u, v_{h}\right)+b\left(v_{h}, p\right)=0, \quad v_{h} \in V_{h}, \\
\left(\frac{d p}{d t}, q_{h}\right)_{Q_{h}}-b\left(u, q_{h}\right)=0, \quad q_{h} \in Q_{h} .
\end{array}\right.
$$

From (5.7), (5.8) and (5.9), we have that at $t=m \tau, m=0,1,2, \ldots$,

(5.10) $\left(D_{\tau} \tilde{u}_{h}, v_{h}\right)_{X_{h}}+v a\left(\tilde{u}_{h}, v_{h}\right)+l\left(\tilde{u}_{h}, v_{h}\right)+m\left(\tilde{u}_{h}, v_{h}\right)+b\left(v_{h}, \tilde{p}_{h}\right)$

$$
\begin{gathered}
=\left(D_{\tau} \tilde{u}_{h}-\frac{d u}{d t}, v_{h}\right)_{X}+l\left(\tilde{u}_{h}-u, v_{h}\right)+m\left(\tilde{u}_{h}-u, v_{h}\right)+b\left(v_{h}, \tilde{p}_{h}-p\right) \\
\text { for } v_{h} \in X_{h},
\end{gathered}
$$

(5.11) $\left(D_{\tau} \tilde{p}_{h}, q_{h}\right)_{Q_{h}}-b\left(\tilde{u}_{h}(t+\tau), q_{h}\right)=\left(\left(D_{\tau}-\frac{d}{d t}\right) p(t), q_{h}\right)_{Q_{h}}-b\left(\tilde{u}_{h}(t+\tau)-u, q_{h}\right)$ for $q_{h} \in Q_{h}$.

There are elements $f_{m} \in X_{h}$ and $g_{m} \in Q_{h}$ such that

(5.12) $\quad\left(f_{m}, v_{h}\right)_{X_{h}}=$ the right hand side of (5.10), for $v_{h} \in X_{h}$,

(5.13) $\quad\left(g_{m}, q_{h}\right)_{Q_{h}}=$ the right hand side of (5.11), for $q_{h} \in Q_{h}$.

Let $v_{m}=\tilde{u}_{h}(m \tau)-u_{h}(m \tau), q_{m}=\tilde{p}_{h}(m \tau)-p_{h}(m \tau)$, and let $T=T_{h}, B=B_{h}$. Then from (5.10), (5.11) and $\left(\Pi_{h, \tau}\right)$, it holds that

$$
\begin{cases}\frac{v_{n}-v_{m}}{\tau}+T v_{m}+B^{*} q_{m}=f_{m}, & \\ \frac{q_{n}-q_{m}}{\tau}-B v_{n}=g_{m}, & n=m+1, m=0,1,2, \ldots,\end{cases}
$$

which is rewritten in the following form $\left(\boldsymbol{F}_{\tau}\right)$.

$$
\left\{\begin{array}{l}
\frac{\boldsymbol{v}_{n}-\boldsymbol{v}_{m}}{\tau}=A_{\tau} \boldsymbol{v}_{m}+f_{m}, \quad n=m+1, m=0,1,2, \ldots, \\
\boldsymbol{v}_{0}=\boldsymbol{v}^{0}
\end{array}\right.
$$

where we use the notations:

$$
\begin{aligned}
& \boldsymbol{v}_{m}=\left(\begin{array}{c}
v_{m} \\
q_{m}
\end{array}\right), \\
& \boldsymbol{f}_{m}=\left(\begin{array}{c}
f_{m} \\
g_{m}+\tau B^{*} f_{m}
\end{array}\right), \\
& \boldsymbol{v}^{0}=\left(\begin{array}{c}
\Pi_{h} u_{0}-P_{h} u_{0} \\
0
\end{array}\right),
\end{aligned}
$$


and $\boldsymbol{A}_{\tau}$ is defined by (4.10). Using $\boldsymbol{T}_{h}(t)=\left(1+\tau \boldsymbol{A}_{\tau}\right)^{[t / \tau]}$, the solution $\boldsymbol{v}_{n}$ of $\left(\boldsymbol{F}_{\tau}\right)$ is expressed as

$$
v_{n}=T_{h}(n \tau) v_{0}+\tau \sum_{j=0}^{n-1} T_{h}((n-j-1) \tau) f_{j}
$$

Under the condition (3.10), $\left\|\boldsymbol{T}_{h}(n \tau)\right\|_{\boldsymbol{x}_{h}} \leq 1$. Hence it holds

$$
\left\|\boldsymbol{v}_{n}\right\|_{\boldsymbol{x}_{h}} \leq\left\|\boldsymbol{v}_{0}\right\|_{\mathbf{x}_{h}}+T \max _{0 \leq j \leq n-1}\left\|\boldsymbol{f}_{j}\right\|_{\boldsymbol{x}_{h}}, \quad 0 \leq n \tau \leq T .
$$

From (3.2) and (4.1), there is a constant $c_{0}$ such that

$$
\left\|v_{0}\right\|_{\mathbf{x}_{h}} \leq c_{0} h\left|u_{0}\right|_{2} \text {. }
$$

From (3.2), (5.12), (5.13) and (5.15), there is a constant $c_{1}$ such that

$$
\left\|\boldsymbol{f}_{j}\right\|_{\mathbf{X}_{h}} \leq c_{1}\{h+\tau\} \cdot F,
$$

where $F=\max _{0 \leq t \leq T}\left|\frac{d^{2} u}{d t^{2}}(t)\right|_{0}+\max _{0 \leq t \leq T}\left|\frac{d u}{d t}(t)\right|_{0}+\max _{0 \leq t \leq T}|u(t)|_{2}$

$$
+\max _{0 \leq t \leq T}\left|\frac{d^{2} p}{d t^{2}}(t)\right|_{0}+\max _{0 \leq t \leq T}|p(t)|_{1} .
$$

It is noted that $\left\|\tau B^{*}\right\|_{L(X, Q)}$ is bounded uniformly in $h$ under the condition (3.10) as was shown in Section 4. From (5.17), (5.18), (5.19), we have for $\tilde{\boldsymbol{u}}_{h}=\left(\begin{array}{c}\tilde{u}_{h} \\ \tilde{p}_{h}\end{array}\right)$

$$
\left\|\tilde{\boldsymbol{u}}_{h}(t)-\boldsymbol{u}_{h}(t)\right\|_{\boldsymbol{x}_{h}} \leq c_{0}\left|u_{0}\right|_{2} h+c_{1} T F\{h+\tau\} \quad \text { for } t \in[0, T] .
$$

By a standard argument, we can show that there is a constant $c_{2}$ such that

$$
\left\|\boldsymbol{u}(t)-\tilde{\boldsymbol{u}}_{h}(t)\right\|_{\boldsymbol{X}} \leq c_{2}\{h+\tau\} F, \quad \text { for } \quad t \in[0, T] .
$$

The conclusion (5.5) follows from (5.20) and (5.21).

\section{References}

[1] Fichera, G., Existence theorems in elasticity, Handbuch der Physik VI a, Mechanics of Solid II, Springer, Berlin, (1972), 347-389.

[2] Fujita, H., On the semi-discrete finite element approximation for the evolution equation $u_{t}+A(t) u=0$ of parabolic type, in Miller, J. J. H. (ed), Topics in Numerical Analysis, 3, Academic Press, London, (1977), 143-157.

[3] Fujita, H. and Mizutani, A., On the finite element method for parabolic equations I; approximation of holomorphic semi-groups, J. Math. Soc. Japan, 28 (1976) 749-771 .

[4] Kanayama, H. and Ohtsuka, K., Finite element analysis on the tidal current and COD distribution in Mikawa Bay, Coastal Engineering in Japan, 21 (1978), 157-171.

[5] Kanayama, H. and Ushijima, T., On the viscous shallow-water equations I -derivation and conservation laws-Memoirs of Numerical Mathematics, 8/9 (1982), 39-64. 
[6] _ A mathematical analysis of a finite element scheme for a linearized viscous shallow-water system, in Kawai, T. (ed), Finite element flow analysis, Proc. of the 4th International Symposium on Finite Element Methods in Flow Problems, in Tokyo, July, 1982, Univ. of Tokyo Press, Tokyo, 1982.

[7] - On the viscous shallow-water equations II - a finite element scheme for a linearized system-, in preparation.

[8] Kato, T., Remarks on pseudo-resolvents and infinitesimal generators of semi-groups, Proc. Japan Acad., 35 (1959), 467-468.

[9] — Perturbation theory for linear operators, Springer, Berlin, 1966.

[10] Kikuchi, F. and Ushijima, T., On finite element methods for convection dominated phenomena, Math. Meth. in the Appl. Sci., 4 (1982), 98-122.

[11] Krein, S. G., Linear Differential Equations in Banach Space, (Russian original, Nauka, Moscow, 1967, Japanese translation, Yoshioka-Shoten, Kyoto, 1972).

[12] Okamoto, H., On the semi-discrete finite element approximation for the nonstationary Stokes equation, Jour. Fac. Sci., Univ. Tokyo Sec. IA 29 (1982).

[13] Richtmeyer, R. D. and Morton, K. W., Difference method for initial-value problems, second edition, John Wiley \& Sons, New York, 1967.

[14] Suzuki, T., An abstract study of Galerkin's method for the evolution equation $u_{t}+$ $A(t) u=0$ of parabolic type with the Neumann boundary condition, Jour. Fac. Sci., Univ. Tokyo Sec. IA 25, (1978) 25-46.

[15] Temam, R., Navier-Stokes Equations; Theory and Numerical Analysis, rev. ed. NorthHolland, Amsterdam, 1979.

[16] Trotter, H. F., Approximation of semi-groups of operators, Pacific J. Math., 8 (1958), 887-919.

[17] Ushijima, T., Approximation theory for semi-groups of linear operators and its application to approximation of wave equations, Japan. J. Math. NS, 1 (1975), 185-224.

[18] — On the finite element type approximation of semi-groups of linear operators, in Fujii, H., Kikuchi, F., Nakagawa, T., Ushijima, T. (eds), Numerical Analysis of Evolution equations, Lecture Notes in Numerical and Applied Analysis, 1, Kinokuniya, Tokyo, 1979.

[19] — Note on the error estimate for the Newmark- $\beta$ method applied to the second order linear evolution equation of hyperbolic type, Memoir of Numer. Math., 7 (1980), 53-66.

[20] Yosida, K., Functional Analysis, Springer, Berlin, 1965.

[21] Zlamal, M., Curved elements in the finite element method, I, SIAM J., Numer. Anal., 10 (1973), 229-240. 